\title{
Prevalence and antibiotic susceptibility patterns of Shigella and Salmonella among children aged below five years with Diarrhoea attending Nigist Eleni Mohammed memorial hospital, South Ethiopia
}

Wondwossen Abebe ${ }^{1,3^{*}}$, Alemu Earsido ${ }^{2}$, Solomon Taye ${ }^{3}$, Mesfin Assefa ${ }^{3}$, Adane Eyasu ${ }^{3}$ and Girma Godebo ${ }^{3}$

\begin{abstract}
Background: Diarrhoeal disease is the second leading cause of death among children aged below 5 years. Even though, both preventable and treatable diseases, globally there are nearly 1.7 billion cases of childhood diarrhoeal disease and responsible for killing around 525,000 children every year. Shigella and Salmonella species were the leading cause of etiologic agents for diarrhoea associated deaths. The aim of this study was to determine the prevalence and antibiotic susceptibility patterns of Shigella and Salmonella isolated from children aged below 5 years with diarrhoea attending Nigist Eleni Mohammed Memorial Hospital, Hossana, South Ethiopia.

Methods: A cross sectional study was conducted from June 02 to September 24, 2017. Two hundred four children aged below 5 years with diarrhoea were enrolled consecutively using convenience sampling technique. Stool specimens were processed in accordance with the standard bacteriological methods and antibiotic susceptibility pattern of the isolates was determined using disc diffusion method. Data were analyzed using SPSS version 20.

Results: Out of the 204 children aged below 5 years with diarrhoeal disease 19/204 (9.3\%, [95\%Cl, 5.7-13.7\%]) of them were positive for bacterial growth, of which 17/204(8.3\%) were Shigella species and 2/204(1\%) were Salmonella species. Both Shigella and Salmonella isolates were $100 \%$ susceptible to norfloxacin, nalidixic acid and kanamycin. However, isolates of Shigella showed 100, 76.5 and $64.7 \%$ resistance to ampicillin, gentamicin and cotrimoxazole respectively while Salmonella species were highly resistant to ampicillin and gentamicin (100\% each).

Conclusions: Salmonella and Shigella species is prevalent in the current study area. Among the tested antibiotics, norfloxacin, nalidixic acid and kanamycin were found to be most effective for both isolates. Both species are developing resistance to the commonly prescribed antibiotic. Therefore, culture based bacterial species identification and antimicrobial susceptibility testing services are strongly recommended to avoid empirical treatment in the study area.
\end{abstract}

Keywords: Shigella, Salmonella, Diarrhoea, Children aged below 5 years, Antibiotic susceptibility, Hosanna, Ethiopia

\footnotetext{
* Correspondence: wondisweet@gmail.com

'Department of Medical Microbiology, School of Biomedical and Laboratory

Sciences, College of Medicine and Health Sciences, University of Gondar,

Gondar, Ethiopia

${ }^{3}$ Department of Medical Laboratory Science, College of Medicine and Health

Sciences, Wachemo University, Hossana, Ethiopia

Full list of author information is available at the end of the article
}

(c) The Author(s). 2018 Open Access This article is distributed under the terms of the Creative Commons Attribution 4.0 International License (http://creativecommons.org/licenses/by/4.0/), which permits unrestricted use, distribution, and reproduction in any medium, provided you give appropriate credit to the original author(s) and the source, provide a link to the Creative Commons license, and indicate if changes were made. The Creative Commons Public Domain Dedication waiver (http://creativecommons.org/publicdomain/zero/1.0/) applies to the data made available in this article, unless otherwise stated. 


\section{Background}

Diarrhoeal disease is the second leading cause of death among children aged below 5 years. Even though, both preventable and treatable diseases, globally there are nearly 1.7 billion cases of childhood diarrhoeal disease and responsible for killing around 525,000 children every year. The burden of diarrhoeal disease is highest in developing country where there is poor sanitation, inadequate hygiene, unsafe drinking water, as well as poorer overall health and nutritional status [1].

A wide variety of aetiological agents are responsible for causing diarrhea such as Shigella spp (Shigellosis), Vibro cholera (Cholera), typical enteropathogenic Escherichia coli (tEPEC), entrotoxigonic E. coli (ETEC), non-typhoidal Salmonella spp, Clostridium difficile, Aeromonas spp, Campylobacter spp, (Campylobacter enteritis), Rotavirus (Rotaviral enteritis), enteric Adenovirus (serotype 40 and 41), Norovirus, Entamoeba histolytica (Amoebiasis), and Cryptosporidium spp (Cryptosporidiosis). Among these agents Rotavirus, Shigella spp and Salmonella spp are the leading cause of diarrhea deaths [2].

In recent years the emergence and global dissemination of Salmonella and Shigella species resistant to ampicilline, chloramphenicol, tetracycline and co-trimoxazole (trimethoprime-sulphamethoxazole) has been increasingly documented in developing countries [3]. Inappropriate antibiotic use and limited laboratory facilities to test antimicrobial susceptibility has led to an increased antimicrobial resistance and reduced therapeutic efficacy in the developing countries [4].

In Ethiopia, diarrhea is one of the major contributors to deaths for children aged below 5 years and contributes to more than one in every ten $(13 \%)$ child deaths. The prevalence of diarrhoea is high $(12 \%)$ in children aged below 5 years. However, it is relatively higher among children aged from 6 to 23 months [5]. Furthermore, isolates of salmonella and shigella showed different prevalence rates and high rate of drug resistance to the commonly used antibiotic agents in different regions [4, 6-9]. There is no published data from the study area (Hosanna town) on the prevalence and antimicrobial susceptibility patterns of salmonella and Shigella among diarrhoeic children aged below 5 years. Therefore, this study aimed to fill the existing information gap in the current study area.

\section{Methods}

\section{Study design, area and population}

A cross sectional study was carried out from June 2017 to September 2017 on children aged below 5 years with diarrhea attending Nigist Eleni Mohammed Memorial Hospital (NEMMH), Hosanna town, South Ethiopia. Hosanna town is located $232 \mathrm{~km}$ southwest of Addis Ababa, the capital city of Ethiopia. The hospital is located in Hosanna city and is the only hospital in the area. Currently, the hospital provides health services to more than 2.3 million people residing in Hadiya and Kembata Tembaro zones. It has 88 beds for inpatient services in four wards (medical, pediatric, surgical and gynecology/obstetrics). The hospital is also provides outpatient health services. The Hosanna branch of regional health laboratory is also found in the hospital compound providing its services.

\section{Sample size and sampling technique}

The sample size was calculated based on the assumption of $5 \%$ expected margins of error and 95\% confidence interval, taking the prevalence of $15 \%$ from the previous study which was conducted in Butajira town, Ethiopia on diarrheal patients [9] using a single population proportion formula (9) as follows.

$$
\mathrm{n}=\frac{(\mathrm{Z} \alpha / 2)^{2} \mathrm{P}(1-\mathrm{P})}{\mathrm{d}^{2}}
$$

Where:

$\mathrm{n}=$ calculated sample size.

$\mathrm{Z}=$ standard normal deviate at $95 \%$, Confidence Interval $=1.96$.

$\mathrm{P}=$ prevalence from the previous study $=15 \%$.

$\mathrm{d}=$ precision level $=0.05$.

The calculated sample size was 196, adding 5\% non-response rate, the total calculated sample size was 206 study participants. The study participants were enrolled consecutively using convenience sampling technique until a sample size of 206 study participants was achieved. All study participants were affected by diarrhea (diarrhea defined as the passage of three or more loose or liquid stools per day) [1]. The physicians collected socio-demographic information and other required information like antibiotic taken before data collection from their parents/guardians. However, children who had taken antibiotic within 7 days before data collection, those who were aged above 5 years and those children whose parents/guardians did not consent to participate in the study were excluded from the study. The aims of the study and benefits of participation were clearly explained for the participants prior to data collection. Participation was on voluntarily basis and they were told that it was within their right to withdraw from the study at any time in the course of the study.

\section{Specimen collection and cultural identification}

Freshly passed stool was collected, placed immediately in Cary Blair transport medium (Oxoid Ltd., Basingstoke, UK) and then transported to the Hosanna branch of regional laboratory within 3 hours of collection for further processing. The specimens were placed in 
Selenite F enrichment broth (Oxoid, UK) and incubated at $37{ }^{\circ} \mathrm{C}$ for $24 \mathrm{~h}$. And then sub-cultured onto deoxycholate agar (DCA) and xylose lysine deoxycholate agar (XLD,) (Oxoid UK) agar and incubated at $37{ }^{\circ} \mathrm{C}$ for 18 $24 \mathrm{~h}$. The growth of Salmonella and Shigella species was detected by its colony characteristic appearance on XLD agar (Shigella: red colonies, Salmonella: red with a black centre) and DCA (Shigella: pale colonies, Salmonella: black center pale colonies). The suspected colonies were further tested through a series of biochemical tests to identify Shigella and Salmonella species [10].

Disk diffusion technique was performed to assess the antibiotic resistance / susceptibility pattern of Salmonella and Shigella isolates. The antibiotic susceptibility testing of all strains were carried out on Muller-Hinton agar (Oxoid, UK) with antibiotic discs (Oxoid, UK) using the single disc diffusion technique against ampicillin $(10 \mu \mathrm{g})$, co-trimoxazole, (trimethoprim/sulphamethoxazole) 1.25/ $23.75 \mu \mathrm{g})$, chloramphenicol $(30 \mu \mathrm{g})$, ciprofloxacin $(5 \mu \mathrm{g})$, ceftriaxone $(30 \mu \mathrm{g})$, nalidixic acid $(30 \mu \mathrm{g})$, gentamicin $(10 \mu \mathrm{g})$, norfloxacin $(10 \mu \mathrm{g})$ and kanamycin $(30 \mu \mathrm{g})$ based on the Standard Operating Procedure (SOP) adapted from Clinical and Laboratory Standards Institute (CLSI 2017 edition) and results were reported as sensitive, intermediate and resistance. To standardize the inoculums density for a susceptibility test, a BaSO4 turbidity standard, equiva lent to a 0.5 McFarland standard was used strictly following the SOP for the preparation and standardization [11]. Multiple drug resistance is defined as the resistance of an isolate to two and more drugs within one class of drug [9].

\section{Data analysis and interpretation}

Data were entered and analyzed using SPSS version 20 software. Results were presented through graphs and tables. Statistical significance of association was measured by using Chi-square test. A $p$-value $<0.05$ was considered as statistically significant.

\section{Results}

Socio-demographic characteristics

A total of 204 children aged below 5 years with diarrhoea were included in the study. Out of the 204 study participants, 103/204 (50.5\%) were females. The ages of the study participants ranged from 2 to 59 months with a mean of 26.2 months (SD \pm 11.457 ): $88 / 204$ (43.1\%) of them were between 24 and 35 months, 59/204(28.9\%) were between 12 and 23 months, and 29/204(14.2\%) were between 36 and 47 months old (Fig. 1). Two study participants were excluded due to insufficient sample provision for laboratory investigation.

\section{Prevalence of Shigella and Salmonella}

Of the 204 children aged below 5 years with diarrhoeal disease 19/204 (9.3\%, [95\% CI, 5.7-13.7\%]) revealed bacterial growth, of which 17/204(8.3\%) were Shigella species and 2/204(1\%) were Salmonella species. The frequency of isolation of Shigella species was highest among the age group of 24 and 35 months, furthermore, the only two Salmonella isolates were encountered among 12-23 month old, but has no statistical significance with the age interval. Among the 19 culture positive children, 11/19(57.9\%) were females (Table 1).

\section{Antibiotic susceptibility test patterns}

Among all antibiotic tested, all isolates of Shigella spp. were susceptible to norfloxacin $17 / 17(100 \%)$, nalidixic acid 17/17(100\%), and kanamycin 17/17(100\%) while, 14/17(82.4\%) Strains were resistant to ampicilin, 13/ $17(76.5 \%)$ to gentamicin, and $11 / 17(64.7 \%)$ to co-trimo xazole. Salmonella isolates were also susceptible to norfloxacin, nalidixic acid, kanamycin, chloramphenicol, ciprofloxacin and ceftriaxone, but $100 \%$ resistant to gentamicin and ampicillin (Table 2).

The overall multiple drug resistance patterns were 12/ $19(63.2 \%)$ while none of them was sensitive to all

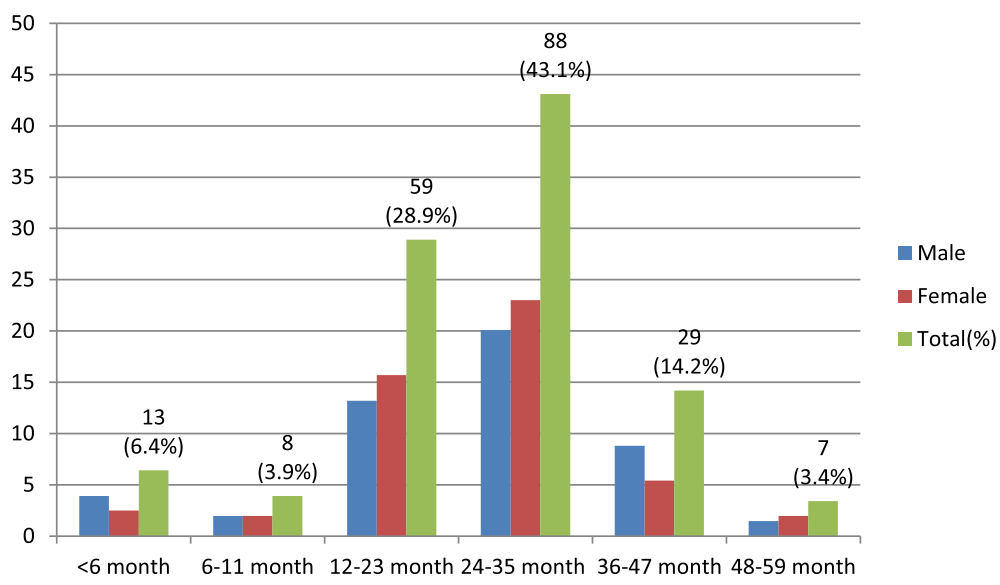

Fig. 1 Distribution of participants by age and sex 
Table 1 Distribution of Shigella and Salmonella by age and sex isolated from children aged below five years with diarrhea at NEMM Hospital, Hosanna town, South Ethiopia, 2017

\begin{tabular}{|c|c|c|c|c|c|c|}
\hline \multirow[t]{2}{*}{ Variable } & \multicolumn{2}{|c|}{ Salmonella spp. $(n=2)$} & \multicolumn{2}{|c|}{ Shigella spp. $(n=17)$} & \multirow{2}{*}{$\begin{array}{l}\text { Total positive } \\
(n=19) \text { No (\%) }\end{array}$} & \multirow[t]{2}{*}{$P$-value } \\
\hline & Positive No (\%) & Negative No (\%) & Positive No (\%) & Negative No (\%) & & \\
\hline \multicolumn{7}{|c|}{ Age(month) } \\
\hline$<6$ & $0(0)$ & $13(100)$ & $0(0)$ & $13(100)$ & $0(0)$ & \multirow[t]{6}{*}{0.181} \\
\hline $6-11$ & $0(0)$ & $8(100)$ & $1(12.5)$ & $7(87.5)$ & $1(5.3)$ & \\
\hline $12-23$ & $2(3.4)$ & 57 (96.6) & $3(5.1)$ & $56(94.9)$ & $5(26.3)$ & \\
\hline $24-35$ & $0(0)$ & $88(100)$ & $10(11.4)$ & 78 (88.6) & $10(52.6)$ & \\
\hline $36-47$ & $0(0)$ & $29(100)$ & $2(6.9)$ & $27(93.1)$ & $2(10.5)$ & \\
\hline $48-59$ & $0(0)$ & $7(100)$ & $1(14.3)$ & $6(85.7)$ & $1(5.3)$ & \\
\hline \multicolumn{7}{|l|}{ Sex } \\
\hline Male & $1(1)$ & $100(99)$ & $7(6.8)$ & $94(91.2)$ & $8(42.1)$ & \multirow[t]{2}{*}{0.811} \\
\hline Female & $1(1)$ & $102(99)$ & $10(9.7)$ & $93(90.3)$ & $11(57.9)$ & \\
\hline
\end{tabular}

antimicrobial drugs tested. Of the seventeen Shigella isolates 11/17 (64.7\%) were resistant to more than 3 antimicrobials, In addition among the two isolates of Salmonella spp. one was found to be resistant to more than 3 antibiotics (Table 3 ).

\section{Discussion}

The isolation rate of Shigella (8.3\%) in our study was comparable to previous studies in Ethiopia: Addis Ababa, 9.1\% [12], Hawassa, 7\% [13], Gondar, 7.5\% [14], 8.7\% [15], Bahir Dar, 9.5\% [16] and Mekelle, 6.9\% [17]. However, it is lower than other findings in Hawassa [5], Jimma [18], Harar [19], Gondar [4] and Bahir Dar [20] with the isolation rate $34.6,20.1,14.6,16.9$ and $14.9 \%$ respectively. Our finding is higher than studies conducted in other part of Ethiopia: Butajira, 4.5\% [9], Jimma, 2.3\% [21], 1.1\% [22] and Gondar, $4.6 \%$ [23]. These variations may be due to differences in study participant, study period and increased awareness of the community about personal and environmental hygiene made by the health extension workers being implemented by the government and by health science students from Wachamo University during their field practice. Furthermore, there is no research conducted around Hosanna town on identification and characterization of Shigella isolates to see prevalence variation over time.
In the current study, the isolation rate of Salmonella (1\%) was found to be lower than other studies done in Ethiopia at different areas: Addis Ababa, 3.95\% [12], Butajira, 4.5\% [9], Harar, 11.5\% [8], Jimma, 15.4\% [18] and Bahir Dar 7.8\% [16]. However, it was consistent with the findings reported in Addis Ababa, 0\% [24], Hawassa $0 \%$ [25], 2.5\% [13], Gondar 1.6\% [26], 1.1\%(23). This could be due to difference in study participant, study period, geographical and seasonal variation, and increased awareness of the community about personal and environmental hygiene. Better awareness of the community especially mothers about their personal and environmental hygiene directly influence the prevalence of Shigella and Salmonella among their children.

Norfloxacin, nalidixic acid and kanamycin were 100\% effective against Shigella while there was a resistance for ampicillin (82.4\%), gentamicin (76.5\%) and co-trimo xazole $(64.7 \%)$ which is comparable with previous report from Gondar, where 100 and $93 \%$ of the isolates were sensitive to norfloxacin and kanamycin respectively [15]. In addition, in similar study conducted in Gondar, Ethiopia, Shigella isolates showed comparable pattern of high resistance against ampicillin (78.9\%) and co-trimo xazole $(84.6 \%)$ and lower resistance to gentamicin (12.2\%), ciprofloxacin (2.2\%) and norfloxacin (1.1\%) [14].These findings indicate that treatment needs to be

Table 2 Antibiotic resistance patterns of Shigella and Salmonella isolate among children aged below 5 years with diarrhoea at NEMM Hospital, Hosanna town, South Ethiopia, 2017

\begin{tabular}{lllllllllll}
\hline Isolate & \multicolumn{1}{l}{ No $(\%)$ of isolates resistance } & & & & \\
\cline { 2 - 10 } & $\mathrm{n}$ & $\mathrm{AMP}$ & $\mathrm{GEN}$ & KAN & NAL & CRO & SXT & CAF & NOR & CIP \\
\hline Shigella & 17 & $14(82.4)$ & $13(76.5)$ & $0(0)$ & $0(0)$ & $3(17.6)$ & $11(64.7)$ & $8(47.1)$ & $0(0)$ & $3(17.6)$ \\
Salmonella & 2 & $2(100)$ & $2(100)$ & $0(0)$ & $0(0)$ & $0(0)$ & $1(50)$ & $0(0)$ & $0(0)$ & $0(0)$ \\
Total & 19 & $16(84.2)$ & $15(78.9)$ & $0(0)$ & $0(0)$ & $3(15.8)$ & $12(63.2)$ & $8(42.1)$ & $0(0)$ & $3(15.8)$
\end{tabular}

Key: AMP Ampicillin, TTC Tetracycline, GEN Gentamicin, KAN kanamycin, NAL Nalidixic acid, CRO ceftiraxone, SXT Trimethoprime-Sulphamethoxazole(co-trimoxazole), CAF Chloramphenicol, NOR Norfoloxacin, CIP Ciprofloxacin 
Table 3 Antibiogram of Shigella and Salmonella isolated among children aged below 5 years with diarrhoea in NEMMH, Hosanna town, South Ethiopia, 2017

\begin{tabular}{llllll}
\hline Isolate & Antibiogram & & & \\
\cline { 2 - 6 } & No. of isolates & $\mathrm{R}_{0}$ No $(\%)$ & $\mathrm{R}_{1}$ No $(\%)$ & $\mathrm{R}_{2}$ No $(\%)$ & $4(26.7)$ \\
\hline Shigella & 17 & $1(5.9)$ & $1(6.2)$ & $1(50)$ & $11(64.7)$ \\
Salmonella & 2 & 0 & 0 & 29.4 & $1(50)$ \\
Total & 19 & 5.3 & 5.6 & 63.2 \\
\hline
\end{tabular}

$R 1$ Resistance for one drug, $R 2$ Resistance for two drugs, $\geq R 3$ Resistance for three and above drugs

based on species identification and susceptibility testing rather than the currently practiced empirical treatment $[8,24]$.

The high level of antibiotic susceptibility of Salmonella to chloramphenicol, ciprofloxacin and norfloxacin is in agreement with earlier studies reported from Ethiopia [9, 21]. The resistance of Salmonella towards ampicillin (100\%) was similar to studies from Jimma, 100\% [22] and Harar, 100\% [8]. High resistance was also observed to gentamaycin $(100 \%)$ which agrees with reports Addis Ababa [4]; in contrast to low resistance report from Jimma 5.2\% [22] and Harar 7.2\% [8]. The rise in resistance might be due to selective pressure created by the use of antimicrobials in food processing animals and irrational use of antibiotics [27].

In present study, over $63 \%(12 / 19)$ of the isolates were resistance two or more antibiotics and none of the strains were sensitive to all antimicrobials tested. Of the seventeen Shigella isolates, 11/17 (64.7\%) were resistant to more than 3 antimicrobials. This shows that antibiotics remain the most important therapy for successful bacterial infections; however these inexpensive and widely available antimicrobials can no longer be used empirically $[3,17]$.

\section{Conclusions}

Salmonella and Shigella species is prevalent in the current study area. Among the tested antibiotics, norfloxacin, nalidixic acid and kanamycin were found to be most effective for both isolates. Both species are developing resistance to the commonly prescribed antibiotic. Therefore, culture based bacterial species identification and antimicrobial susceptibility testing services are strongly recommended to avoid empirical treatment in the study area.

\section{Abbreviations \\ DCA: Deoxycholate Agar; NEMMH: Nigist Eleni Mohammed Memorial Hospital; SOP: Standard Operating Procedure; XLD: Xylose lysine deoxycholate agar}

\section{Acknowledgments}

We would like to thank Hosanna branch of regional laboratory stuff for sharing equipment and laboratory space. Also we are grateful to all the study participants for their willingness.

\section{Funding}

The study was funded by the Wachemo University.
Availability of data and materials

The datasets used and analyzed during the current study are available from the corresponding author on reasonable request.

\section{Authors' contributions}

WA was conceived the study, designed data collection, conducted data analysis and interpretation.AE1, ST, MA and GG, assisted in data collection and supervision. AE1, ST, MA and AE2 interpreted the results and reviewed the initial and final drafts of the manuscript. All authors read and approved the final manuscript.

\section{Ethics approval and consent to participate}

This research was conducted after obtaining ethical clearance from Wachemo University ethical review board. Permissions letter were obtained from the concerned bodies of the Hospital. All underage participants had their written informed consent provided by their parent or legal guardian. For each confirmed infection case, the responsible clinician of the patient was informed and treatment was started as per the guideline. Information obtained in each course of the study was kept confidential.

\section{Consent for publication}

Not applicable

\section{Competing interests}

The authors declare that they have no competing interests.

\section{Publisher's Note}

Springer Nature remains neutral with regard to jurisdictional claims in published maps and institutional affiliations.

\section{Author details}

${ }^{1}$ Department of Medical Microbiology, School of Biomedical and Laboratory Sciences, College of Medicine and Health Sciences, University of Gondar, Gondar, Ethiopia. ${ }^{2}$ Department of Public Health, College of Medicine and Health Sciences, Wachemo University, Hossana, Ethiopia. ${ }^{3}$ Department of Medical Laboratory Science, College of Medicine and Health Sciences, Wachemo University, Hossana, Ethiopia.

Received: 10 January 2018 Accepted: 11 July 2018

Published online: 25 July 2018

\section{References}

1. UNICEF/WHO. Diarrhoea: why children are still dying and what can be done. Geneva: WHO Press; 2009. p. 1-68. Available from http://www.who. int/topics/diarrhoea/en

2. GBD Diarrhoeal Diseases Collaborators. Estimates of global, regional, and national morbidity, mortality, and aetiologies of diarrhoeal diseases: a systematic analysis for the global burden of disease study 2015. Lancet Infect Dis. 2017:17:909-48.

3. Okeke IN, Laxminarayan R, Bhutta Z, et al. Antimicrobial resistance in developing countries. Part I: recent trends and current status. Lancet Infect Dis. 2005:5:481-93.

4. Roma B, Worku S, Mariam ST, Langeland N. Antimicrobial susceptibility pattern of Shigella isolates in Hawassa. Ethiop J Health Dev. 2000;14:149-54.

5. Central Statistical Agency (CSA) [Ethiopia] and ICF. Ethiopia Demographic and Health Survey 2016. Addis Ababa, Ethiopia, and Rockville, Maryland, USA: CSA and ICF; 2016. 
6. Asrat D. Shigella and Salmonella serogroups and their antibiotic susceptibility patterns in Ethiopia. East Mediterr Health J. 2008;14:760-7.

7. Yismaw G, Negeri C, Kassu A. A five-year antimicrobial resistance pattern observed in Shigella species isolated from stool samples in Gondar University hospital, Northwest Ethiopia. EthiopJHealth Dev. 2006; 20(3):194-8.

8. Reda A, Seyoum B, Yimam J, et al. Antibiotic susceptibility patterns of Salmonella and Shigella isolates in Harar, eastern Ethiopia. J Infect Dis Immun. 2011;3:134-9.

9. Mengstu G, Mulugeta G, Lemma T, Aseffa A. Prevalence and antimicrobial susceptibility patterns of Salmonella serovars and Shigella species. J Microb Biochem Technol. 2014;2:1-7.

10. Forbes BA, Sahm DF, Weissfeld AS, editors. Bailey \& Scott's diagnostic Microbiology. 11 th ed. Philadelphia: Mosby Co; 2002. p. 368-75.

11. CLSI. Performance standards for antimicrobial susceptibility testing. 27th ed. CLSI supplement M100. Wayne, PA: Clinical and Laboratory Standards Institute; 2017.

12. Mamuye $Y$, Metaferia G, Birhanu A, Desta K, Fantaw S. Isolation and antibiotic susceptibility patterns of Shigella and Salmonella among under 5 children with acute Diarrhoea: a cross-sectional study at selected public health facilities in Addis Ababa, Ethiopia. Clin Microbiol. 2015;4:186. https://doi.org/10.4172/2327-5073.1000186.

13. Mulatu G, Beyene G, Zeynudin A. Prevalence of Shigella, Salmonella and campylobacter species and their susceptibility patters among under five children with diarrhea in Hawassa town, South Ethiopia. Ethiop J Health Sci. 2014;24(2):101-8.

14. Tiruneh M. Serodiversity and antimicrobial resistance pattern of Shigella isolates at Gondar University teaching hospital, Northwest Ethiopia. Jpn J Infect Dis. 2009;62:93-7.

15. Andualem B, Kassu A, Diro E, Moges F, Gedefaw M. The prevalence and antimicrobial responses of Shigella isolates in HIV-1 infected and uninfected adult diarrhoea patients in north West Ethiopia. Ethiop J Health Dev. 2006; 20(2):99-105.

16. Maritu A, Gebremariam Y, Mulugeta K, Bayeh A, Endalkachew N, Melaku A. Prevalence and antibiogram of Shigella and Salmonella spp. from under five children with acute diarrhea in Bahir Dar town. EJST. 2015;8(1):27-35.

17. Gebrekidan A, Tsehaye A, Getahun K, Araya G. Prevalence and antimicrobial susceptibility patterns of Shigella among acute diarrheal outpatients in Mekelle hospital, northern Ethiopia. BMC Res Notes. 2015;8:611.

18. Mache A. Antibiotic resistance and sero-groups of Shigella among pediatric outpatients in Southwest Ethiopia. East Afr Med J. 2001;78(6): 296-9.

19. Mekonnen $\mathrm{H}$, Kebede $\mathrm{A}$, Menkir S. Isolation rate and drug resistance patterns of Shigella species among diarrheal patients attending at Hiwot Fana hospital, Harar, Ethiopia. Ethiop J Sci Technol. 2014;7(1):15-25.

20. Debas G, Kibret M, Biadglegne F, Abera B. Prevalence and antimicrobial susceptibility patterns of Shigella species at Felege Hiwot referral hospital, Northwest Ethiopia. Ethiop Med J. 2011;49:249-56.

21. Beyene G, Tasew H. Prevalence of intestinal parasite, Shigella and Salmonella species among diarrheal children in Jimma health center, Jimma Southwest Ethiopia: a cross sectional study. Ann Clin Microbiol. 2014;13(1):10.

22. Lamboro T, Ketema T, Bacha K. Prevalence and antimicrobial resistance in Salmonella and Shigella species isolated from outpatients, Jimma University specialized hospital, Southwest Ethiopia. Can J Infect Dis Med Microbiol. 2016;2016:4210760.

23. Demissie AT, Wubie TM, Yehuala FM, Fetene MD, Gudeta AG. Prevalence and antimicrobial susceptibility patterns of Shigella and Salmonella species among patients with diarrhea attending Gondar town health institutions, Northwest Ethiopia. Sci J Pub Health. 2014;2(5): 469-75.

24. Hawaz H, Girma S, Tezera Y, Ahmed U, Kelel M. Prevalence and drug susceptibility pattern of Shigella and Salmonella species in under ten diarrhoeic children admitted to Tirunesh-Beijing hospital. Int J Sci Rep. 2017;3(2):637

25. Desta M, Asrat D, Weldeamanuel Y, Nigusie D. Prevalence of intestinal parasites and Salmonella and Shigella among food handlers at food service establishments in the main campus and health sciences College of Hawassa University, Hawassa, Ethiopia. Ethiop J Health Dev. 2014; 28(1):29-34
26. Huruy K, Kassu A, Mulu A, Worku N, Fetene T, Gebretsadik S, Biadglegne F, Belyhun F, Muche A, Gelaw A, Anagaw A, Yifru S, Wondie Y, Bekele A, Tiruneh M, Reissig D, Moges F. Intestinal parasitosis and shigellosis among diarrheal patients in Gondar teaching hospital, Northwest Ethiopia. BMC Res Notes. 2011;4(1):472.

27. Addisalem HB, Bayleyegn MZ. Tetracycline residue levels in slaughtered beef cattle from three slaughterhouses in Central Ethiopia. J Global Vet. 2012;8(6): 546-54.

\section{Ready to submit your research? Choose BMC and benefit from:}

- fast, convenient online submission

- thorough peer review by experienced researchers in your field

- rapid publication on acceptance

- support for research data, including large and complex data types

- gold Open Access which fosters wider collaboration and increased citations

- maximum visibility for your research: over $100 \mathrm{M}$ website views per year

At BMC, research is always in progress.

Learn more biomedcentral.com/submissions 